\title{
A NOTE ON THE CONCORDANCE HOMOTOPY GROUP OF REAL PROJECTIVE SPACE
}

H. SCHNEIDER AND R. WELLS

\begin{abstract}
By means of the mapping torus construction the following theorem is proved

THEOREM. If $r \equiv 3 \bmod 4$ and $r \geqslant 7$, and $\mathscr{\Phi}_{r}$ is a homotopy $P_{r}$, then there is an isomorphism $\pi_{0}$ Diff $^{+}: \mathscr{P}_{r} \cong \pi_{0}$ Diff $^{+}: P_{r}$.
\end{abstract}

1. Introduction. For a manifold $M$, let $\operatorname{Diff}^{+}(M)$ be the group of diffeomorphisms of $M$ isotopic to diffeomorphisms leaving some nonempty open set fixed. Let $\pi_{0}$ Diff $^{+}: M$ denote that group factored by those concordant to the identity. Similarly, let $\operatorname{Diff}^{+}(M, A)$ denote the subgroup of $\operatorname{Diff}^{+}(M)$ of diffeomorphisms fixing $A$, and let $\pi_{0} \operatorname{Diff}^{+}:(M, A)$ denote $\operatorname{Diff}^{+}(M, A)$ factored by the subgroup of those concordant $\bmod A$ to the identity.

Let $P_{r}$ denote real projective space of dimension $r$. In [4], an author establishes an isomorphism $\pi_{0} \operatorname{Diff}^{+}: P_{r} \cong \pi_{r+1+k}\left(P_{\infty} / P_{k}\right)$ for $r$ $\equiv 11 \bmod 16$ and $k=\alpha 2^{L}-r-1$ with $\alpha$ a positive integer and $L$ a large positive integer. Suppose $M$ is a smooth closed, $(l-1)$-connected and oriented manifold of dimension $n$ with $l=[n / 2]$. Suppose $\zeta: M \rightarrow M$ is a free smooth involution; then there is an equivariant embedding $\left(S^{l},-1\right) \subset(M, \zeta)$ producing an embedding $P_{l} \subset M / \zeta$, and $v(M / \zeta) \mid P_{l}=k \eta_{l}$ where $\eta_{l} \in \widetilde{K O}\left(P_{l}\right)$ is the reduced canonical line bundle. The integer $k$ is well defined $\bmod c(l)$, where $c(l)$ is the order of $K O\left(P_{l}\right)$, and its class $\bmod c(l)$ is called the type of $\zeta$. For $k$ and $l$ even, let

$$
I_{2 l}(k)=\left\{(M, \zeta) \mid M \sim S^{l} \times S^{l}, \text { type } \zeta=k\right\} / \approx,
$$

where $\sim$ means homotopy equivalent, and $\approx$ means orientation-preserving equivariantly diffeomorphic. From [3] we recall that $I_{2 l}(k)$ has a canonical group structure, and that for $l \equiv 6 \bmod 8$ and $k \equiv-2 l \bmod c(l)$ we have an isomorphism $\pi_{2 l+k}\left(P_{\infty} / P_{k-1}\right) \cong I_{2 l}(k)$. Thus we have for such $l$ and $k$ the isomorphism $\pi_{0}$ Diff $^{+}: P_{2 l-1} \cong I_{2 l}(k)$. We will say that a homotopy $P_{r}$ is a smooth closed $r$-manifold $\mathscr{P}_{r}$ homotopy equivalent to $P_{r}$. It is the object of this note to find a generalization, for $\mathcal{P}_{r}$ a homotopy $P_{r}$ with $r \equiv 3 \bmod 4$, of the isomorphism $\pi_{0}$ Diff: $P_{2 l-1} \equiv I_{2 l}(k)$ above.

Received by the editors May 9, 1975 and, in revised form, May 5, 1976.

AMS (MOS) subject classifications (1970). Primary 57D50, 57D65, 57D90, 57E25. 
In [1], the first author introduces abelian groups $I_{n}(k)$ generalizing the $I_{2 l}(k)$ above. Suppose $M$ is a smooth closed $n$-manifold homotopy equivalent to $S^{[n / 2]} \times S^{[(n+1) / 2]}$ and suppose $\zeta: M \rightarrow M$ is a smooth free involution. Let $l=[n / 2]$. We will say $(M, \zeta)$ is admissible if there exist disjoint copies, $P, P^{\prime} \subset M / \zeta$ of $P_{l}$ such that $P^{\prime} \subset M / \zeta-P$ is a homotopy equivalence. With $n$ even, all free involutions are admissible, but when $n$ is odd some are excluded. Then set

$$
I_{n}(k)=\left\{(M, \zeta) \mid M \sim S^{[n / 2]} \times S^{[(n+1) / 2]} \text {, type } \zeta=k, \zeta \text { admissible }\right\} / \approx .
$$

The equivalence relation $\approx$ is the same as before when $k$ is even-orientation preserving equivariant diffeomorphism-but when $k$ is odd, it is only equivariant diffeomorphism. From [1] we recall that $I_{n}(k)$ has a canonical abelian group structure, provided $n \geqslant 6$; also from [1] we recall that there is an exact sequence of abelian groups

$$
\cdots \rightarrow \mathfrak{E}_{n+1}\left(Z_{2},(-1)^{k}\right) \stackrel{\partial}{\rightarrow} I_{n}(k) \stackrel{P}{\rightarrow} \Omega_{n}(\lambda(l, k)) \stackrel{\sigma}{\rightarrow} \mathfrak{E}_{n}\left(Z_{2},(-1)^{k}\right),
$$

where $\mathcal{E}_{j}\left(Z_{2},(-1)^{k}\right)$ is a certain quotient of the Wall surgery group $L_{j}\left(Z_{2},(-1)^{k}\right)$, and $\Omega_{n}(\lambda(l, k))$ is a certain Lashof cobordism group. It follows, for example, that $I_{n}(k)$ is finitely generated.

Now we can state the main theorem of this note.

THEOREM 2. If $r \equiv 3 \bmod 4$ and $r \geqslant 7$, and $\mathscr{P}_{r}$ is a homotopy $P_{r}$, then there is an isomorphism $\pi_{0}$ Diff $^{+}: \mathscr{P}_{r} \cong I_{r+1}(k)$ where $k \equiv-r-1 \bmod c(l)$.

COROLlaRY. If $\mathcal{P}_{r}$ is a homotopy $P_{r}, r \equiv 3 \bmod 4$ and $r \geqslant 7$, then $\pi_{0}$ Diff $^{+}$: $\mathscr{P}_{r} \cong \pi_{0}$ Diff $^{+}: P_{r}$.

The theorem is an immediate consequence of the following theorem. If $\mathscr{P}_{r}$ is a homotopy $P_{r}$, there is an embedding, for $m=[r / 2]$, unique up to isotopy $P_{m-1} \subset \mathscr{P}_{r}$ such that $\pi_{1}\left(P_{m-1}\right) \rightarrow \pi_{1}\left(\mathscr{P}_{r}\right)$ is an epimorphism. Let $N_{r}$ be a tubular neighborhood of $P_{m-1}$ in $\mathscr{P}_{r}$. Let $f: \pi_{0} \operatorname{Diff}^{+}:\left(\mathcal{P}_{r}, N_{r}\right) \rightarrow \pi_{0} \operatorname{Diff}^{+}: \mathscr{P}_{r}$ be the forgetful homomorphism. Then we have the following:

THEOREM 1. Let $r \geqslant 5$ and let $\Phi_{r}$ be a homotopy $P_{r}$. Then there is a homomorphism $\tau: \pi_{0} \operatorname{Diff}^{+}:\left(\mathscr{P}_{r}, N_{r}\right) \rightarrow I_{r+1}(k)$, where $k \equiv-r-1 \bmod c(l)$, such that:

(1) kernel $(\tau) \subset$ kernel $(f)$,

(2) $\tau($ kernel $(f)) \subset \partial \mathfrak{L}_{r+2}\left(Z_{2},(-1)^{k}\right)$,

(3) $\tau$ is an epimorphism.

We continue to use the notation implicit above: Given $r$, we set $l$ $=[(r+1) / 2], m=[r / 2], c(l)=\operatorname{order} \widetilde{K O}\left(P_{l}\right), k=$ class of $-r-1 \bmod c(l)$, and $\eta_{s}=$ canonical line bundle over $P_{s}$. If $(M, N)$ is a smooth manifold pair, $v(N: M)$ denotes the normal bundle of $N$ in $M ; \tau(M)$ denotes the tangent bundle of $M$. If $\mathscr{P}_{r}$ is a homotopy $P_{r}$ we have again the embedding $P_{m-1} \subset \mathscr{P}_{r}$ and its tubular neighborhood $N_{r} \subset \mathscr{P}_{r}$. Since $\mathscr{P}_{r}$ is necessarily tangentially 
homotopy equivalent to $P_{r}$ and since $r-(m-1)=l+1>(m-1)+1$, we have that $N_{r}$ is a smooth embedding of the cell bundle associated with $(l+1) \eta_{m-1}$. There is an obvious homomorphism $\pi_{0} \operatorname{Diff}^{+}:\left(\mathscr{\Phi}_{r}, N_{r}\right)$ $\rightarrow^{f} \pi_{0} \mathrm{Diff}^{+}: \Phi_{r}$.

To see that $f$ is an epimorphism we introduce a homomorphism $d: \pi_{0}$ Diff $^{+}$: $\mathscr{P}_{r} \rightarrow Z_{2}$ defined as follows: If $x \in \pi_{0}$ Diff $^{+}: \mathscr{P}_{r}$, we may choose a representative $\varphi: \mathscr{P}_{r} \rightarrow \mathscr{P}_{r}$ of $x$ such that $\varphi$ fixes $P_{1}$ where $P_{1} \subset P_{m-1} \subset \mathscr{P}_{r}$. Then $d \varphi: v\left(P_{1}: \mathscr{P}_{r}\right) \rightarrow v\left(P_{1}: \mathscr{P}_{r}\right)$ represents a well-defined element $d(x) \in \widetilde{K O}^{-1}\left(P_{1}\right)$ $=Z_{2}$, and $x \rightarrow d(x)$ is a homomorphism.

Proposition 1. $d: \pi_{0}$ Diff $^{+}: \mathscr{P}_{r} \rightarrow Z_{2}$ is trivial.

Proof. We are indebted for the proof to R. Z. Goldstein. As in the definition of $d$, let $\varphi$ represent $x$, such that $\varphi$ fixes $P_{1}$. Let $g \in H^{1}\left(S^{1}: Z_{2}\right)$ and $g^{\prime} \in H^{1}\left(P_{1}: Z_{2}\right)$ be the nontrivial elements. Let $x(\varphi) \in H^{1}\left(S^{1} \times P_{1}: Z_{2}\right)$ be 0 if $d(x)=0$ and $\operatorname{pr}_{1}^{*} g$ if $d(x)=1$. Let $y=\operatorname{pr}_{2}^{*} g^{\prime}$. Let $S^{1} \times_{\varphi} \Phi_{r}$ be the mapping torus of $\varphi$. Then $S^{1} \times P_{1} \subset S^{1} \times_{\varphi} \mathscr{P}_{r}$ and we have that the StiefelWhitney class

$$
\omega\left(v\left(S^{1} \times P_{1}: S^{1} \times_{\varphi} \mathscr{P}_{r}\right)\right)=(1+x(\varphi))(1+y)^{r-1} .
$$

On the other hand, $\varphi$ is homotopic to the identity, so $S^{1} \times_{\varphi} \mathscr{P}_{r}$ has the homotopy type of $S^{1} \times P_{r}$ and $\omega\left(\tau\left(S^{1} \times \mathscr{P}_{r}\right) \mid S^{1} \times P_{1}\right)=(1+y)^{r+1}$. Since $\tau\left(S^{1} \times P_{1}\right)$ is trivial,

$$
\omega\left(\tau\left(S^{1} \times_{\varphi} \mathscr{P}_{r}\right) \mid S^{1} \times P_{1}\right)=\omega\left(\nu\left(S^{1} \times P_{1}: S^{1} \times_{\varphi} \mathscr{P}_{r}\right)\right),
$$

and we get $(1+x(\varphi))(1+y)^{r-1}=(1+y)^{r+1}$, from which $x(\varphi)=0$ follows since $y^{2}=0$. The proposition is proved.

If $x \in \pi_{0}$ Diff $^{+}: \mathscr{P}_{r}$, then there is a representative $\varphi$ that fixes $P_{m-1} \subset \mathscr{P}_{r}$. We would like to find a representative that fixes $N_{r}$. The representative $\varphi$ at most twists $N_{r}$ by an element $d^{\prime}(\phi) \in \widetilde{K O^{-1}}\left(P_{m-1}\right)$.

Proposition 2. $f: \pi_{0} \operatorname{Diff}^{+}:\left(\mathscr{P}_{r}, N_{r}\right) \rightarrow \pi_{0} \mathrm{Diff}^{+}: \mathscr{P}_{r}$ is an epimorphism.

Proof. The map $\widetilde{K O^{-1}}\left(P_{m-1}\right) \rightarrow \widetilde{K O^{-1}}\left(P_{1}\right)$ carries $d^{\prime}(\varphi) \rightarrow d(x)$. This map is an isomorphism for $m \neq \equiv \bmod 4$, so we are done in that case by Proposition 1. If $m \equiv 0 \bmod 4$, then $\widetilde{K O}^{-1}\left(P_{m-1}\right) \equiv \widetilde{K O}^{-1}\left(P_{1}\right)$ is onto with infinite cyclic kernel. Then $d^{\prime}(\varphi) \neq 0$ implies that $v\left(S^{1} \times P_{m-1}: S^{1} \times_{\varphi} \mathscr{P}_{r}\right)$ has a nontrivial rational Pontrjagin class in dimension $m$, which is impossible, and the proposition is proved.

Now we construct the homomorphism $\tau: \pi_{0} \operatorname{Diff}^{+}:\left(\Phi_{r}, N_{r}\right) \rightarrow I_{r+1}(k)$ for $r \geqslant 5$. Briefly, it is the mapping torus construction followed by 'surgery' of $S^{1} \times N_{r} \cup S_{+}^{1} \times \mathscr{P}_{r}$. We construct a smooth manifold triad $\left(X ; \partial_{0} X, \partial_{1} X\right)$ such that $\partial X=\partial_{0} X \cup \partial_{1} X$ and $\partial \partial_{0} X=\partial \partial_{1} X=\partial_{0} X \cap \partial_{1} X$ as follows: $X=D^{2} \times \mathscr{P}_{r}$. With $S_{+}^{1}$ and $S_{-}^{1}$ the right and left hemicircles, respectively, we set $\Gamma=$ closure $\left(\mathscr{P}_{r}-N_{r}\right)$, and $\partial_{0} X=S_{-}^{1} \times \Gamma$, and $\partial_{1} X=S^{1} \times N_{r} \cup S_{+}^{1}$ $\times P_{r}$. 
Now, if $x \in \pi_{0} \operatorname{Diff}^{+}:\left(\mathscr{P}_{r}, N_{r}\right)$ is represented by $\varphi$, the mapping torus $S^{1} \times_{\varphi} \mathscr{P}_{r}$ contains a codimension 0 submanifold canonically isomorphic to $S^{1} \times N_{r} \cup S_{+}^{1} \times \mathscr{P}_{r}$. Thus we may construct a well-defined ' surgery' with $\left(X ; \partial_{0} X, \partial_{1} X\right)$ in place of the usual $\left(D^{r+1} ; S^{l} \times D^{m}, D^{l+1} \times S^{m-1}\right)$ : Set $Y$ $=\left(\left(S^{1} \times_{\varphi} \mathscr{P}_{r}\right) \times[0,1]\right) \cup X$ where $\partial_{1} X$ is identified with $\left(S^{1} \times N_{r} \cup S_{+}^{1} \times \mathscr{P}_{r}\right)$ $\times 1$ by means of the canonical diffeomorphism. Then $\partial Y=\partial_{0} Y \amalg \partial_{1} Y$ with $\partial_{0} Y=\left(S^{1} \times_{\varphi} \mathscr{P}_{r}\right) \times 0$ and $\partial_{1} Y$ the other component of $\partial Y$. It is routine to check that $\partial_{1} Y$ is the orbit manifold of a representative of an element $\tau(\varphi) \in I_{r+1}(k)$. This element $\tau(\varphi)$ is well defined. If $\varphi^{\prime}$ is another representative of $x$, there is a concordance fixed on $N_{r}$ from $\varphi$ to $\varphi^{\prime}$. Constructing $Y^{\prime}$ for $\varphi^{\prime}$ as above, and a similar manifold for the concordance, we obtain an $h$-cobordism finally from $\partial_{1} Y$ to $\partial_{1} Y^{\prime}$ so that $\tau\left(\varphi^{\prime}\right)=\tau\left(\varphi^{\prime}\right)$. Thus the map $\tau: \pi_{0} \operatorname{Diff}^{+}:\left(\mathscr{P}_{r}, N_{r}\right) \rightarrow I_{r+1}(k)$ is well defined by $\tau(x)=\tau(\varphi)$ for $\varphi$ a representative of $x$.

To see that $\tau$ is a homomorphism, we describe $\tau$ a different way. Recall from [1] that the orbit space $Q$ of an element of $I_{r+1}(k)$ is obtained by gluing two copies of $E\left(m \eta_{l}+1\right)$ (the cell bundle associated with $\left.m \eta_{l}+1\right)$ by means of a diffeomorphism $\varphi^{\prime}: \partial E\left(m \eta_{l}+1\right) \rightarrow \partial E\left(m \eta_{l}+1\right)$. Since an $(m+1)$-plane bundle over $P_{l}$ admitting a nonzero section and stably equivalent to $m \eta_{l}$ is uniquely determined up to bundle equivalence, we have a diffeomorphism $\Gamma \times 0 \cup \Gamma \times 1 \cong \partial E\left(m \eta_{l}+1\right)$ where $\partial \Gamma \times 0$ is glued to $\partial \Gamma \times 1$ by the identity. We have obvious homomorphisms

$$
\begin{aligned}
\pi_{0} \operatorname{Diff}^{+}:\left(\mathscr{P}_{r}, N_{r}\right) \rightarrow \pi_{0} \operatorname{Diff}^{+}:(\Gamma, \partial \Gamma) & \rightarrow \pi_{0} \operatorname{Diff}^{+}:(\Gamma \times 0 \cup \Gamma \times 1, \Gamma \times 1) \\
& \rightarrow \pi_{0} \operatorname{Diff}^{+}: E\left(m \eta_{l}+1\right) .
\end{aligned}
$$

If $x$ is represented by $\varphi$, and $\varphi \rightarrow \varphi^{\prime}$ under the above composition, it is straightforward to check that $E\left(m \eta_{l}+1\right) \times 0 \cup_{\varphi^{\prime}} E\left(m \eta_{l}+1\right) \times 1$ represents $\tau(x)$. Thus we have the commutative diagram:

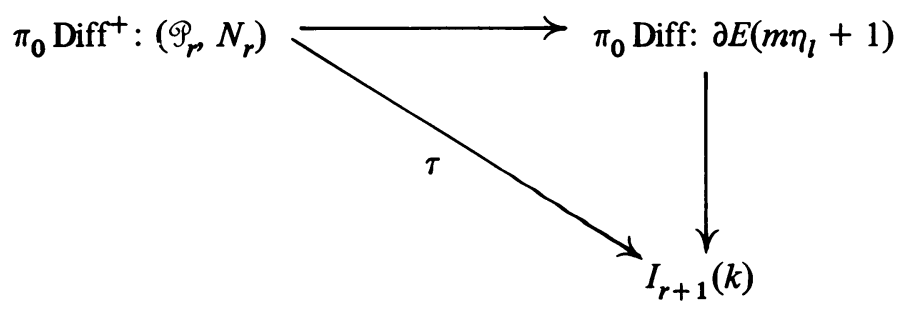

But the horizontal map is already a homomorphism, and according to [1] the vertical map is a homomorphism onto. It follows that $\tau$ is a homomorphism.

Propositron 3. kernel $\tau \subset \operatorname{kernel} f$.

Proof. Suppose $\tau(x)=0$ with $\varphi$ a representative of $x$. Then $\tau(\varphi)$ has orbit space $\partial E\left(m \eta_{l}+2\right)=\partial E$. Then we have $S^{1} \times_{\varphi} \mathscr{P}_{r}=\partial(Y \cup E)$, where $E$ is glued to $Y$ along $\partial Y_{1}=\partial E$. We have $S_{+}^{1} \times \mathscr{P}_{r} \subset \partial(Y \cup E) \subset Y \cup E$, and 
this composition of inclusions is a homotopy equivalence. Using an embed$\operatorname{ding} S_{+}^{1} \times \mathscr{P}_{r} \times[0,1] \subset Y \cup E$ given by a boundary collar, an easy application of the relative $h$-cobordism theorem, as in [4], shows that there is a diffeomorphism $\left(S^{1} \times \mathscr{P}_{r}, 1 \times \mathscr{P}_{r}\right) \cong\left(S^{1} \times \mathscr{P}_{r}, 1 \times \mathscr{P}_{r}\right)$, which is the identity on the relative part. It follows that $f(x)=0$, and Proposition 3 is proved.

Proposition 4. $\tau$ is an epimorphism.

Proof. Suppose $z \in I_{r+1}(k)$ has orbit space $Q$. We know $Q=E\left(m \eta_{l}+1\right)$ $\cup_{\varphi^{\prime}} E\left(m \eta_{l}+1\right)$ for some diffeomorphism $\varphi^{\prime}: \partial E\left(m \eta_{l}+1\right) \rightarrow \partial E\left(m \eta_{l}+1\right)$; also $S_{+}^{1} \times \Gamma=\partial_{0} X$ is diffeomorphic to $E\left(m \eta_{l}+1\right)$. Thus, the triad $\left(X ; \partial_{0} X\right.$, $\left.\partial_{1} X\right)$ determines a surgery $Y$ from $\partial_{1} Y=Q$ to $\partial_{0} Y$. It is routine to check that $\partial_{0} Y \cong S^{1} \times{ }_{\varphi} \mathscr{P}_{r}$ (e.g. as in [4]). By Proposition 2, we may take $\varphi \in \pi_{0}$ Diff: $\left(\mathscr{P}_{r}, N_{r}\right)$, and clearly $\tau(\varphi)=y$. Proposition 4 is proved.

Proof of TheOREM 2. We need only to check that

$$
\tau(\operatorname{kernel} f) \subset \partial \mathcal{L}_{r+2}\left(Z_{2},(-1)^{k}\right)
$$

Recall the definition of the Lashof cobordism group appearing in the exact sequence of [1]. First, $P[l, k] \rightarrow^{\lambda(l, k)} B O$ is a fibration such that $P_{\infty} \rightarrow P[l, k)$ $\rightarrow^{\lambda(l, k)} B O$ is the $l$ th Moore-Postnikov factorization of a map $P_{\infty} \rightarrow B O$ classifying $k \eta_{\infty}$. Then $\Omega_{r+1}(\lambda(l, k))$ is the $(r+1)$ st Lashof cobordism group defined by the fibration $\lambda(l, k)$. The map $p: I_{r+1}(k) \rightarrow \Omega_{r+1}(\lambda(l, k))$ is defined as follows: If $z \in I_{r+1}(k)$ has orbit space $Q$, and $P \subset Q$ is one of the canonical embeddings of $P_{l}$ in $Q$, then there is a commutative diagram

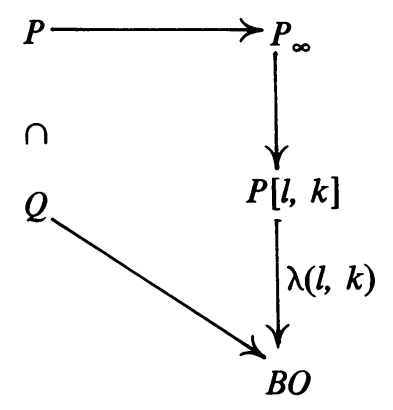

with $Q \rightarrow B O$ a Gauss map. The obstructions are zero to finding a unique lift $\bmod P_{\infty}$ of $Q$ to $P[l, k]$. This lift represents an element of $\Omega_{r+1}(\lambda(l, k))$ which is well defined to be $p(z)$.

Let $x \in$ kernel $(f)$ have representative $\varphi$ and let $\tau(x)=z \in I_{r+1}(k)$, and let $Y$ be the cobordism from $S^{1} \times_{\varphi} \mathscr{P}_{r}$ to $Q$, the orbit space of $z$. Since $x \in$ kernel $f$, we have a diffeomorphism $S^{1} \times \mathscr{P}_{r} \cong S^{1} \times \mathscr{P}_{r}=\partial\left(D^{2} \times \mathscr{P}_{r}\right)$. Gluing $D^{2} \times \mathscr{P}_{r}$ to $Y$ via this diffeomorphism we obtain a manifold $\Lambda$, which may be written $\Lambda=\left(D^{2} \times \mathscr{P}_{r}\right) \times 0 \cup_{\alpha}\left(D^{2} \times \mathscr{P}_{r}\right) \times 1$ with gluing map an embedding $\alpha$ : $\left(S^{1} \times N_{r} \cup S_{+}^{1} \times \mathscr{P}_{r}\right) \times 1 \subset\left(S^{1} \times \mathscr{P}_{r}\right) \times 0$ such that $\alpha((t, \xi), 1)$ $=((t, \xi), 0)$ for $t \in S_{+}^{1}$. We consider the lifting problem set by the following diagram: 


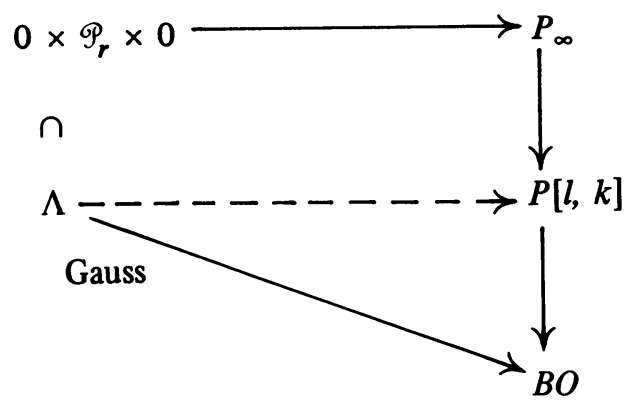

It can be solved iff the lifting problem set by the following diagram can be solved:

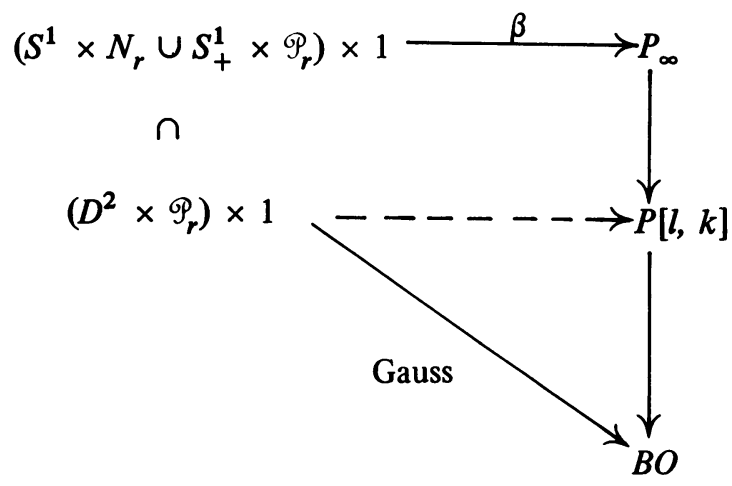

where $\left(S^{1} \times N_{r} \cup S_{+}^{1} \times \mathscr{P}_{r}\right) \times 1 \rightarrow^{\beta} P_{\infty}$ is $\alpha$ followed successively by projection $\left(S^{1} \times \mathscr{P}_{r}\right) \times 0 \rightarrow \mathscr{P}_{r}$ and then $\mathscr{P}_{r} \rightarrow P_{\infty}$. But $\varphi \in \operatorname{Diff}^{+} \mathscr{P}_{r}$ implies $\varphi$ homotopic to the identity so there is a homotopy commutative diagram:

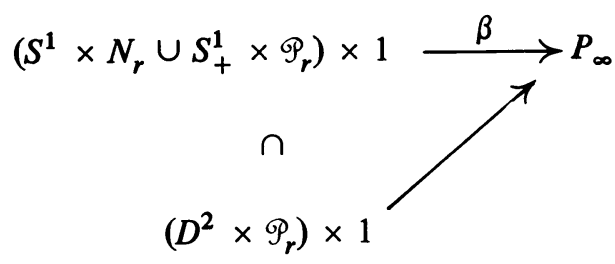

From this diagram follows the solution of the second lifting problem, and so of the first. Let $v: \Lambda \rightarrow P[l, k]$ be that solution. Then $v \mid Q$ represents $p(z)$, and thus $0=p(z)=p(\tau(x))$. From the exact sequence of [1], it follows that $\tau(x) \in \partial \mathcal{L}_{r+2}\left(Z_{2},(-1)^{k}\right)$, the proof of Theorem 2 is complete.

Theorem 1 is an immediate consequence of Theorem 2 and the fact that $\mathfrak{L}_{s}\left(z_{2},+1\right)=0$ for $s \equiv 1 \bmod 4[2]$.

\section{BIBLIOGRAPHY}

1. H. W. Schneider, Groups of free involutions of homotopy $S^{[n / 2]} \times S^{[(n+1) / 2]}$ s, Trans. Amer. Math. Soc. 206 (1975), 99-136.

2. C. T. C. Wall, Surgery on compact manifolds, Academic Press, New York, 1970. 
3. Robert Wells, Free involutions of homotopy $S^{l} \times S^{l}$ s, Illinois J. Math. 15 (1971), 160-184. MR 42 \#6838.

4. - The concordance diffeomorphism group of real projective space, Trans. Amer. Math. Soc. 192 (1974), 319-337. MR 49 \#3986.

Department of Mathematics, Roosevelt University, Chicago, Ilunois 60605

Department of Mathematics, Pennsylvania State University, University Park, PennsyyVANIA 16802 